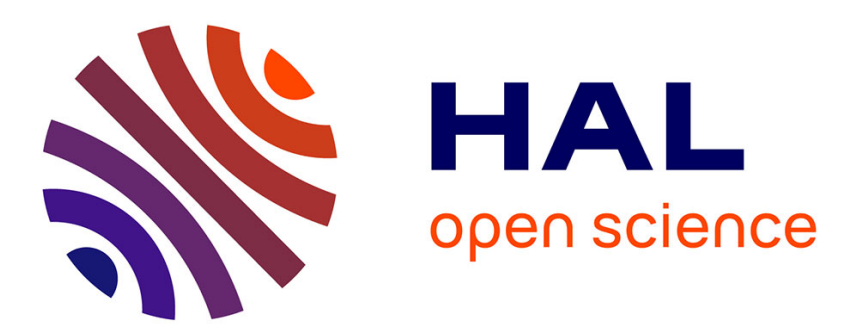

\title{
Complex Mode Spectra of Graphene-Based Planar Structures for $\mathrm{THz}$ Applications
}

Walter Fuscaldo, P. Burghignoli, P. Baccarelli, A. Galli

\section{To cite this version:}

Walter Fuscaldo, P. Burghignoli, P. Baccarelli, A. Galli. Complex Mode Spectra of Graphene-Based Planar Structures for THz Applications. Journal of Infrared, Millimeter and Terahertz Waves, 2015, 36 (8), pp.720-733. 10.1007/s10762-015-0178-0 . hal-01165595

\section{HAL Id: hal-01165595 https://hal-univ-rennes1.archives-ouvertes.fr/hal-01165595}

Submitted on 19 Jun 2015

HAL is a multi-disciplinary open access archive for the deposit and dissemination of scientific research documents, whether they are published or not. The documents may come from teaching and research institutions in France or abroad, or from public or private research centers.
L'archive ouverte pluridisciplinaire HAL, est destinée au dépôt et à la diffusion de documents scientifiques de niveau recherche, publiés ou non, émanant des établissements d'enseignement et de recherche français ou étrangers, des laboratoires publics ou privés. 


\title{
Complex Mode Spectra of Graphene-based Planar Structures for $\mathrm{THz}$ Applications
}

\author{
W. Fuscaldo ${ }^{1,2}$, P. Burghignoli ${ }^{1}$, P. Baccarelli ${ }^{1}$, and A. Galli ${ }^{1}$ \\ ${ }^{1}$ Department of Information Engineering, Electronics and \\ Telecommunications,, Sapienza University of Rome, 00184 Rome, \\ Italy \\ ${ }^{2}$ Institut d'Électronique et de Télécommunications de Rennes \\ (IETR), UMR CNRS 6164, Université de Rennes 1, 35042 Rennes, \\ France
}

\begin{abstract}
In this work we analyze in detail the modal properties of a graphenebased planar waveguide (GPW) in the THz frequency range. The structure consists of a graphene sheet placed on top of a grounded dielectric slab. As is known, the surface conductivity of the graphene sheet can easily be tuned with a bias voltage via electric-field effect; we show here how such a bias affects the propagation features of both TM and TE modes supported by the GPW. An extensive dispersion analysis is performed for complex modes in both guided and radiative (leaky) regimes, considering also dielectric and metal losses as well as nonlocal effects in graphene. In particular, we focus on the behavior of the fundamental leaky modes since they exhibit quite interesting radiation features for suitable values of the bias. These results are very promising for the development of reconfigurable leaky-wave Fabry-Perot cavity antennas based on graphene at $\mathrm{THz}$ frequencies.
\end{abstract}

\section{Introduction}

The pioneering experiments on graphene led by A. K. Geim and K. S. Novoselov [1] have paved the way to the application of this promising material in a multitude of scenarios including integrated technologies, especially at terahertz $(\mathrm{THz})$ frequencies, i.e., that part of the spectrum that nominally goes from $300 \mathrm{GHz}$ to $3 \mathrm{THz}[2]$. In this frequency range graphene shows interesting properties, because its surface conductivity (which completely characterizes its electromagnetic properties, due to the mono-atomic layer structure) becomes mostly reactive [3] and hence can support plasmonic propagation. Moreover, surface plasmon polariton (SPP) waves supported by graphene may have a guided 
wavelength much shorter than the wavelength of plane waves in free space, thus resulting in a tight transverse confinement of the SPP field [4], especially if compared to SPP propagation along ordinary metal surfaces. However, the most intriguing feature of graphene is perhaps the possibility of dynamically tuning its conductivity through the application of an electrostatic bias field, which lays the ground for the development of reconfigurable $\mathrm{THz}$ devices.

Despite all these excellent properties graphene was initially considered mainly as an alternative to post-silicon transistors [5] rather than as a material for antennas and other passive devices. Specifically in antenna applications, graphene was first employed as a parasitic layer of a $\mathrm{THz}$ dipole antenna array radiating around $1 \mathrm{THz}[6]$. Also the propagation of SPPs along an infinite graphene sheet was theoretically derived [3], [7] and the first study on graphene used as an actual antenna radiator was presented in [8]. There, it was shown that graphene allows for the realization of miniaturized resonant and reconfigurable $\mathrm{THz}$ antennas with good efficiency, compared to the small electrical size, and good direct matching, as required by most of the present $\mathrm{THz}$ communication and sensing devices. Over the years, other works [9], [10] have further investigated the possibilities of graphene in antenna design.

All these works consider the radiation mechanism through the excitation of a transverse-magnetic (TM) SPP; to our knowledge only two very recent works [11], [12] show the possibility of designing a graphene-based waveguide through the excitation of leaky modes [13], [14]. In [11], a sheet of graphene is sinusoidally modulated by applying a DC bias to a polysilicon layer located beneath it, in order to control its surface reactance; the pointing angle and the leakage rate can thus be dynamically varied by modifying the bias, allowing for electronic beam scanning at a fixed operating frequency. In [12], a 2D-periodic graphenebased high-impedance surface is used to design a 2D leaky-wave antenna with reconfigurable radiation pattern; however, no information is provided on the dispersion properties of the involved leaky modes.

To the best of our knowledge, in the literature a detailed dispersion analysis is not yet available which considers the propagation of all the possible modes existing in a multilayer structure comprising a graphene layer, both in bound regimes (surface waves, SWs, and in particular SPPs) and in radiative regimes (leaky waves, LWs). Such an investigation would be extremely helpful to clarify how the frequency-dependent behavior of graphene surface conductivity affects modal propagation, especially at $\mathrm{THz}$ frequencies where its variation is not negligible, and even more when a voltage bias is applied in order to modify the graphene chemical potential. With reference to guidance phenomena, a complete modal analysis would furnish fundamental information for the development of graphene-based planar waveguides (GPWs). In particular, structures that are constituted by a dielectric substrate entirely covered at the bottom by a metal ground plane opaque to radiation and on top by a graphene sheet whose reflectance may be controlled through a bias voltage can be considered as reference structures for the design of novel graphene-based Fabry-Perot cavity antennas (FPCAs) [15]. In this framework, the waveguide can be fed by a simple, nondirective source (e.g., a slot etched in the ground plane and back-illuminated 
by a coherent $\mathrm{THz}$ source) in order to excite a pair of weakly attenuated leaky modes that propagate along the structure and mainly determine its radiation features through their complex propagation constants [13]-[15].

In this work we deal with a complete dispersion analysis of a GPW within the band of 0.25-2.0 THz. For the sake of simplicity we start from an ideal structure [3] consisting of a dielectric-filled parallel-plate waveguide (PPW) whose upper plate is made of graphene. A detailed dispersion analysis is performed by numerically solving the relevant dispersion equation obtained through the transverse-resonance technique [16]-[19]. The main result of this analysis is represented by the fact that for suitably high values of the chemical potential the first higher-order modes exhibit leaky regimes with low attenuation constant, hence they may be employed to achieve scannable directive beams in graphenebased tunable FPCAs [20].

\section{Graphene-based Planar Waveguides and their Dispersion Analysis}

The structure of the GPW considered here is shown in Fig. 1 along with the relevant transverse equivalent network (TEN) [16]-[19]. It consists of a dielectricfilled PPW where the lower plate is assumed first a perfectly electric conducting (PEC) plane, whereas the upper plate is a graphene plane that acts as a partially reflecting sheet (PRS) in order to allow for radiation and exhibit reconfigurability properties. A polysilicon layer is used as a gate electrode to control graphene conductivity, but it is safely neglected in the equivalent transmissionline model due to its extremely thin profile [11]. In this idealized structure we have assumed several simplifying hypotheses. In particular, we have considered a two-dimensional structure independent of $y$ and laterally infinite, in which the ground plane is treated as an ideal conductor characterized by an infinite value of the conductivity and the substrate has no dielectric losses. A more realistic model will be treated in Section 4, where it will be shown that the introduction of realistic losses does not affect significantly the results of our analysis.

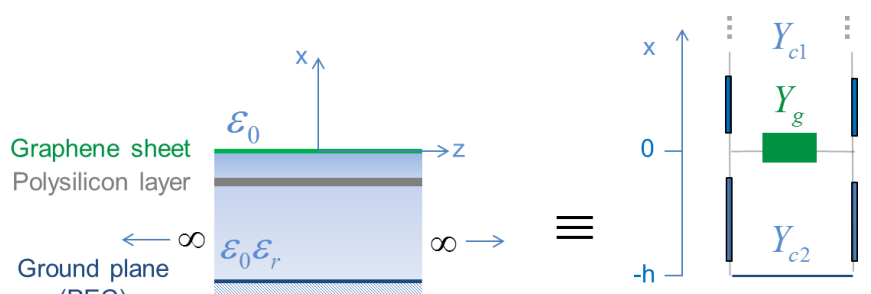

(PEC)

Figure 1: 2D-section of the structure under analysis and its transverse equivalent network (TEN). The polysilicon layer has been safely excluded from the TEN due to its extreme thinness. 


\subsection{Graphene conductivity and biasing}

As is known, graphene conductivity depends on several parameters and it can be derived in scalar form (i.e., neglecting nonlocal effects) through Kubo formula [21]. In the low $\mathrm{THz}$ band and at room temeperature it is usually sufficient to take into account only the intraband contributions, which give rise to a Drudelike dispersion behavior:

$$
\sigma=-\frac{j q_{e}^{2} k_{B} T}{\pi \hbar^{2}\left(\omega-j \tau^{-1}\right)^{2}}\left(\frac{\mu_{c}}{k_{B} T}+2 \ln \left\{\exp \left[-\mu_{c} /\left(k_{B} T\right)\right]+1\right\}\right)
$$

where $k_{B}$ is the Boltzmann constant, $\hbar$ is the reduced Planck constant, $-q_{e}$ is the electron charge, $\mu_{c}$ is the chemical potential, $T$ is the temperature, and $\tau$ is the relaxation time (for all numerical results we have used $T=300 \mathrm{~K}$, and $\tau=3 \mathrm{ps})$.

Clearly, $\sigma$ is strongly affected by the values of $\mu_{c}$ (see Fig. 2) which is in turn related to the electrostatic bias $E_{0}$ through [22]

$$
\begin{aligned}
& E_{0}=\frac{q_{e}}{\pi \varepsilon_{0} \hbar^{2} v_{F}^{2}} \int_{0}^{\infty} \mathcal{E}\left[f_{d}(\mathcal{E})-f_{d}\left(\mathcal{E}+2 \mu_{c}\right)\right] d \mathcal{E} \\
& f_{d}(\mathcal{E})=\left(1+\exp \left[\left(\mathcal{E}-\mu_{c}\right) /\left(k_{B} T\right)\right]\right)^{-1}
\end{aligned}
$$

where $\varepsilon_{0}$ is the vacuum permittivity, $v_{F}$ is the Fermi velocity (for graphene $v_{F} \simeq 10^{6} \mathrm{~m} / \mathrm{s}$ ), and $\mathcal{E}$ is the energy. $E_{0}$ has been calculated for different discrete values of $\mu_{c}$ in the range $0-1 \mathrm{eV}$ by numerically solving the integral on the right-hand side of the equation; as shown in [11] and depending on the biasing scheme, it results that the maximum absolute value of the chemical potential that can be obtained with practical values of the DC bias voltage is around $1 \mathrm{eV}$, which corresponds through Eq. (2a) to $E_{0} \simeq 7.5 \mathrm{~V} / \mathrm{m}$.

It should be mentioned that in recent years more sophisticated models for graphene conductivity have been derived, which take into account its non-local

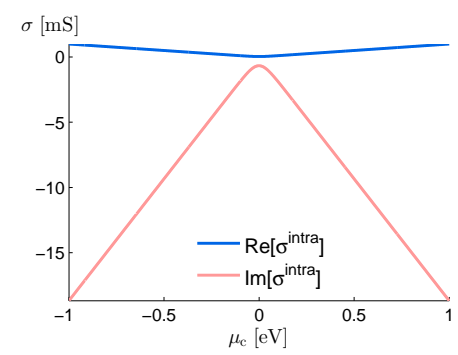

(a)

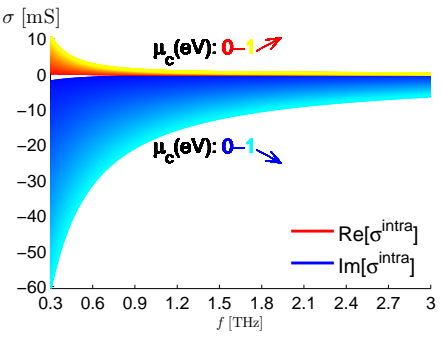

(b)

Figure 2: (a) Graphene surface conductivity vs. chemical potential in the range -1 to $1 \mathrm{eV}$ at the frequency of $1 \mathrm{THz}$. (b) Graphene surface conductivity vs. frequency in the band $0.3-3 \mathrm{THz}$ for chemical potential raising from 0 to $1 \mathrm{eV}$. 
(i.e., spatially dispersive) nature and thus require a dyadic description [23], [24]. These have also been considered in this investigation; however, it will be shown that, for the considered ranges of parameters, non-local effects have a negligible impact on dispersion and radiation properties.

\subsection{Dispersion analysis}

The dispersion equation for modes propagating along the considered GPW is obtained by enforcing the condition of resonance on the relevant transverse equivalent network (see Fig. 1), where $Y_{g}=\sigma$ and the equivalent admittances in air $Y_{c 1}$ and inside the slab $Y_{c 2}$ for TE and TM modes have the following expressions:

$$
\begin{aligned}
Y_{c 1}^{\mathrm{TM}} & =\frac{\omega \varepsilon_{0}}{k_{x 1}} & Y_{c 2}^{\mathrm{TM}} & =\frac{\omega \varepsilon_{0} \varepsilon_{r}}{k_{x 2}} \\
Y_{c 1}^{\mathrm{TE}} & =\frac{k_{x 1}}{\omega \mu_{0}} & Y_{c 2}^{\mathrm{TE}} & =\frac{k_{x 2}}{\omega \mu_{0}}
\end{aligned}
$$

where $k_{x 1}=\sqrt{k_{0}^{2}-k_{z}^{2}}$ and $k_{x 2}=\sqrt{k_{0}^{2} \varepsilon_{r}-k_{z}^{2}}$ are the transverse wavenumbers in air and in the dielectric, respectively, $k_{0}$ is the free-space wavenumber, and $k_{z}=\beta_{z}-j \alpha_{z}$ is the complex longitudinal wavenumber, with $\beta_{z}$ and $\alpha_{z}$ the relevant phase and attenuation (or leakage) constants, respectively.

The resulting dispersion equation for TE modes is

$$
\sqrt{1-\hat{k}_{z}^{2}}+\sigma \eta_{0}-j \sqrt{\varepsilon_{r}-\hat{k}_{z}^{2}} \cot \left(k_{0} h \sqrt{\varepsilon_{r}-\hat{k}_{z}^{2}}\right)=0
$$

whereas for TM modes is

$$
\left(\sqrt{1-\hat{k}_{z}^{2}}\right)^{-1}+\sigma \eta_{0}-j \varepsilon_{r}\left(\sqrt{\varepsilon_{r}-\hat{k}_{z}^{2}}\right)^{-1} \cot \left(k_{0} h \sqrt{\varepsilon_{r}-\hat{k}_{z}^{2}}\right)=0
$$

where $\eta_{0}$ is the free-space impedance and $\hat{k}_{z}=k_{z} / k_{0}$ is the normalized longitudinal wavenumber. A key point in the numerical solution of Eq. (5) and Eq. (6) is the choice of the determination of the square root that defines the vertical wavenumber $k_{x 1}$ [13], [25]. In fact, whereas the corresponding choice for the square root that defines the wavenumber in the slab $k_{x 2}$ is immaterial (because the dispersion equations are even functions of $k_{x 2}$ ) the two determinations of $k_{x 1}$ provide different dispersion equations. As is customary in the study of leaky modes, we distinguish between proper modes with $\operatorname{Im}\left[k_{x 1}\right]<0$ (hence modal fields that tend exponentially to zero at infinity in the transverse direction) and improper modes, with $\operatorname{Im}\left[k_{x 1}\right]>0$ (whose modal fields instead increase exponentially at infinity in the transverse direction) [13], [25].

\subsection{Complex mode dispersion}

In this section numerical results will be reported that illustrate the modal spectrum of the GPW shown in Fig. 1 in the range $0.25-2 \mathrm{THz}$ and for different 
bias conditions. The waveguide is assumed to be filled with a dielectric medium with $\varepsilon_{r}=3.8$ (quartz) and thickness $h \approx 77 \mu \mathrm{m} \approx\left(\lambda / 2 \sqrt{\varepsilon_{r}}\right)$ at $f=1 \mathrm{THz}$. The obtained dispersion behaviors are then compared with those of the relevant grounded dielectric slab (GDS), where the graphene sheet has been removed, and with those of a standard PPW, where the graphene sheet has been substituted by a perfectly conducting metal plate.

In order to obtain the sought modal spectra, the roots of Eq. (5) and Eq. (6) have been found by means of a numerical technique using a Padé-based rootfinding algorithm [26], that is an exponentially convergent procedure that allows for an efficient computation of the complex propagation wavenumbers for layered structures. One of the key aspects of the algorithm lies in a correct choice of the initial points. Since graphene at low $\mathrm{THz}$ frequencies is a rather bad conductor, the unbiased graphene-based PW (henceforth referred to as GPW) can be seen as a perturbation of the GDS, and the proper real solutions of the GDS dispersion relation can be used as good initial guess points for the relevant proper complex solutions in the (lossy) GPW. In fact, proper complex solutions corresponding to surface waves of the GPW are characterized by very low values of the attenuation constant. Results shown in Fig. 3 confirm how the dispersion curves of the GPW are only slightly different from those of the GDS. As can be seen, in both the TE and TM cases the propagating modes exhibit the well-known transition regions between bound and leaky regimes typical of dielectric-based open guiding structures [27], [28]. Unfortunately, LWs of the GPW are not suitable for efficient radiation here, because they exhibit a very high leakage rate [13] especially at low frequency, where the leaky-wave cutoff condition $\left(\hat{\beta}_{z}=\hat{\alpha}_{z}\right)$ is met [20]. One way to obtain reasonable values of the leakage rate is to increase the surface susceptance. As stated in section 2.1, this can be achieved by increasing the chemical potential of graphene (see Fig. 2(b)). Since the most interesting results have been obtained for $\mu_{c}=1 \mathrm{eV}$ we will refer to this value when we consider the biased graphene-based PW (BGPW). As expected, in both TE and TM cases the dispersion curves of BGPW in Fig. 3 are shifted closer to the PPW ones.

Dispersion curves of TE and TM fundamental modes for three different values of $\mu_{c}=0,0.2,1 \mathrm{eV}$ are shown in Fig. 4 in order to describe in more detail the nature of improper and proper complex solutions in lossy GPW and to highlight the effect of increasing the bias. As concerns improper leaky modes, we note that the introduction of a (lossy) graphene sheet causes the generation of an improper complex pair of solutions, the leaky-wave solution, i.e., with $\alpha_{x}<0$ and $\alpha_{z}>0$ (see red dashed lines in Fig. 4), and one with no physical meaning, i.e., $\alpha_{x}<0$ and $\alpha_{z}<0$ (see blue dashed lines in Fig. 4) [13]. The leaky-wave solution is physical when $0<\hat{\beta}_{z}<1$ and can hence contribute to radiation, if properly excited. We remind that the leaky-wave solution corresponds to a pole of the Green's function of the waveguide: the LW is physical when the pole is captured by the relevant steepest-descent path (SDP) [13]. Furthermore, as concerns the nonphysical improper complex solution, by increasing frequency it becomes proper complex and reaches the cutoff condition of the corresponding 


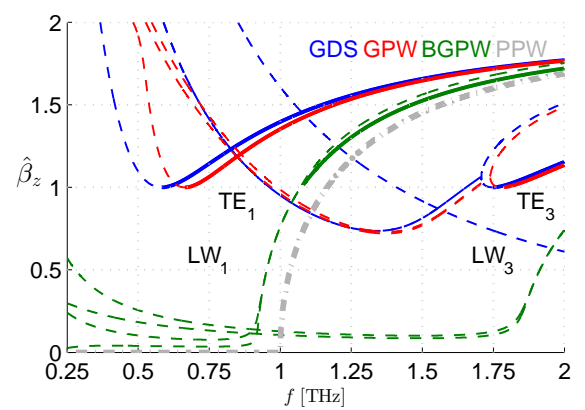

(a)

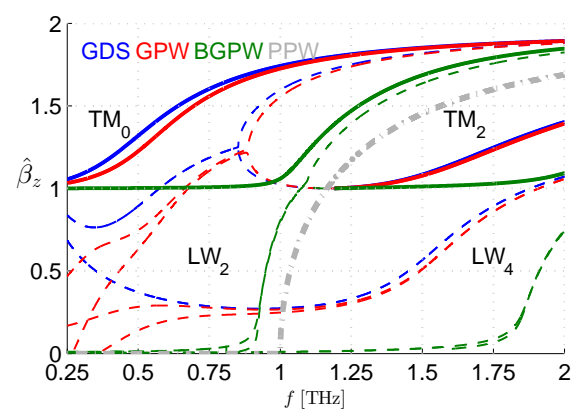

(c)

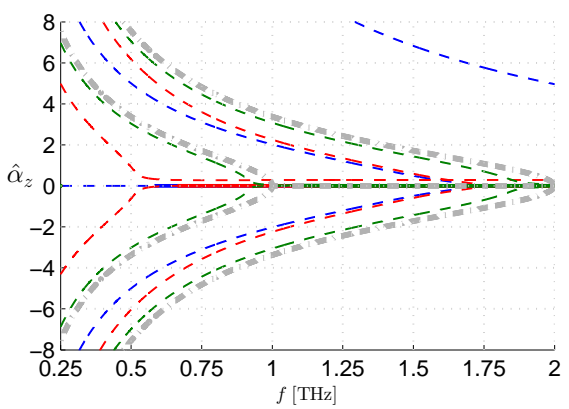

(b)

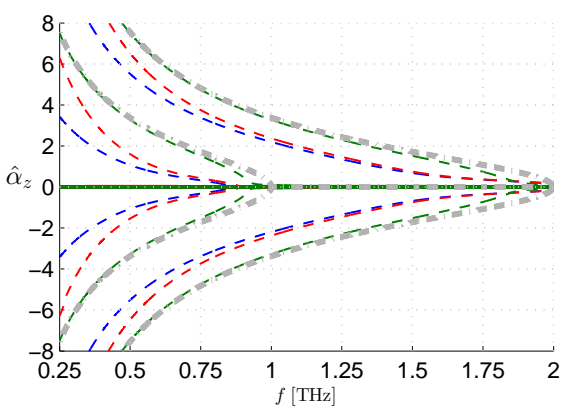

(d)

Figure 3: Dispersion curves of SWs and LWs within the band $0.25-2 \mathrm{THz}$ for a GDS (blue lines), a GPW (red lines), a BGPW (green lines), and a PPW (gray lines). In dashed lines improper waves, in solid lines proper waves. In (a) $\hat{\beta}_{z}$, in (b) $\hat{\alpha}_{z}$ for TE modes, and in (c) $\hat{\beta}_{z}$, in (d) $\hat{\alpha}_{z}$ for TM modes.

surface waves (see blue solid lines in Fig. 4). This kind of evolution of the complex wavenumbers below cutoff is common to all the SW higher-order modes in both TE and TM cases. Finally, specific attention is devoted to the behavior of the fundamental TE improper complex solution of the GPW (the $\mathrm{TE}_{1}$ mode) as $\mu_{c}$ increases. As is well known, for the fundamental TE mode of the GDS (blue dashed line in Fig. 3), there exists only a real improper pole below cutoff (whereas for the high-order modes a complex pair exists). However, we observe in Fig. 4 that as $\mu_{c}$ increases up to $1 \mathrm{eV}$ the fundamental TE improper complex solution with $\alpha_{z}<0$ gradually joins that with $\alpha_{z}>0$ (a solution that never exists in a GDS and that is of no interest in a GPW). Furthermore, the latter becomes a fast (and physical) leaky mode in the frequency range $0.5-0.75 \mathrm{THz}$.

The main results introduced by the application of the bias are better shown in Fig. 5 where the dispersion curves for the fundamental TE and TM LWs have been reported in (a) for three significant values of the chemical potential as frequency varies from 0.75 to $1.1 \mathrm{THz}$, and in (b) for a fixed frequency $(f=0.92$ 


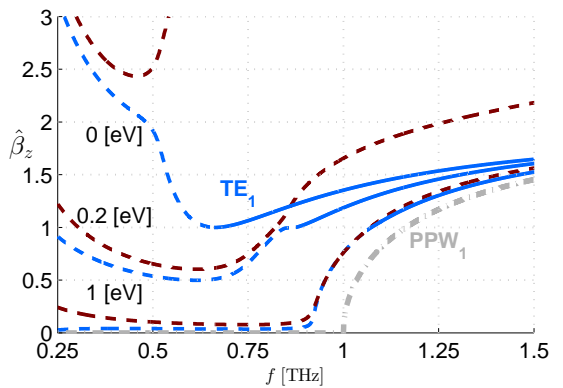

(a)

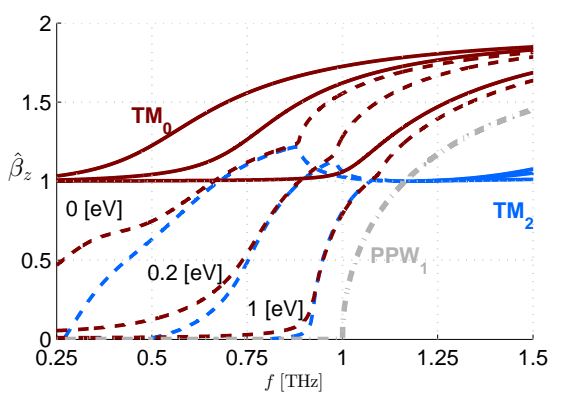

(c)

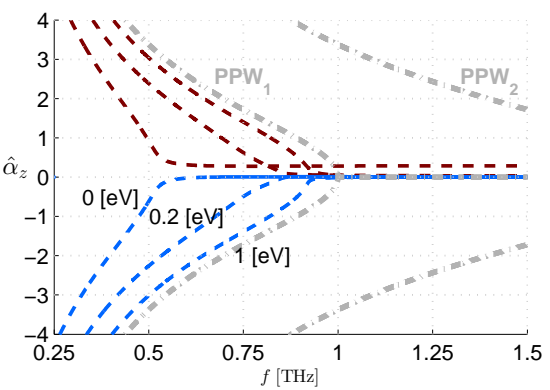

(b)

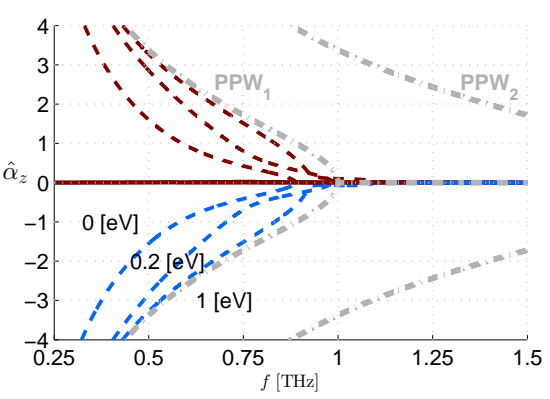

(d)

Figure 4: Dispersion curves of SWs and LWs within the band 0.25-1.5 THz for three different values of the chemical potential. In dashed red line improper leaky poles, in dashed blue line improper non-physical poles, in solid red line SWs for the fundamental TM mode and in solid blue line the remaining SWs. In (a) $\hat{\beta}_{z}$, in (b) $\hat{\alpha}_{z}$ for TE modes, and in (c) $\hat{\beta}_{z}$, in (d) $\hat{\alpha}_{z}$ for TM modes.

$\mathrm{THz}$ ) as chemical potential varies from 0.2 to $1 \mathrm{eV}$. We recall here that the main features of the field radiated by a LW are simply related to the normalized LW wavenumber $\hat{k}_{z}=\hat{\beta}_{z}-j \hat{\alpha}_{z}$. In particular, above the $\mathrm{LW}$ cutoff where $\beta_{z}=\alpha_{z}$, the angle of maximum radiation $\theta_{\mathrm{M}}$ is given by $\sin \theta_{\mathrm{M}} \simeq \sqrt{\hat{\beta}_{z}^{2}-\hat{\alpha}_{z}^{2}}$, which takes the customary form $\sin \theta_{\mathrm{M}} \simeq \hat{\beta}_{z}$ when $\beta_{z} \gg \alpha_{z}$, hence the variation of the phase constant with frequency or other parameters is associated with beam scanning; on the other hand, the half-power beam width $\Delta \theta$ of the main radiated lobe is proportional to the normalized attenuation constant $\hat{\alpha}_{z}$ [13], [14].

Considering now Fig. 5(a), it is clear that the radiative behavior over frequency associated with the fundamental LWs improves for higher values of $\mu_{c}$ since the leakage rate reaches lower values for both the TE and TM cases. Considering Fig. 5(b), we remark that the frequency of $f=0.92 \mathrm{THz}$ corresponds to the leaky-wave cutoff condition for both TE and TM LWs when a bias of $1 \mathrm{eV}$ is applied (as can be seen by inspection of Fig. 5(a)). Here, the beam 


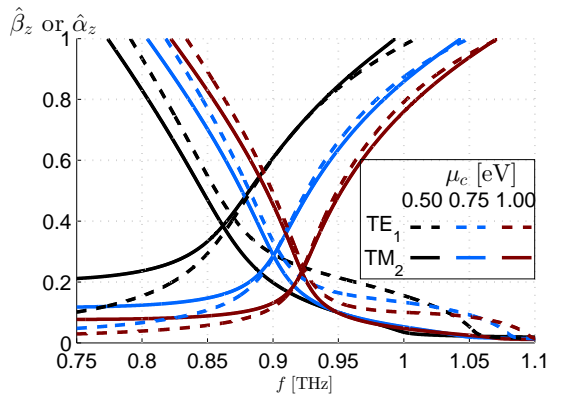

(a)

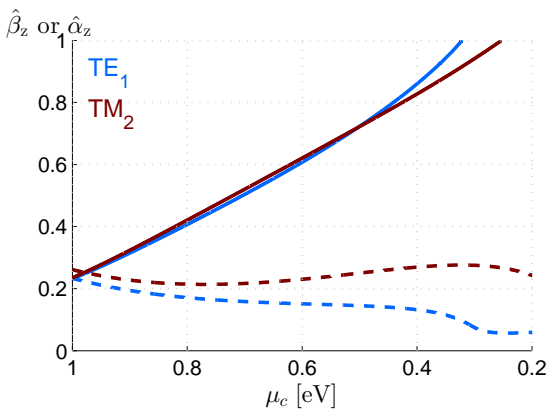

(b)

Figure 5: Dispersion curves of the $\mathrm{TE}_{1}, \mathrm{TM}_{2}$ fundamental LWs (a) within the band $0.75-1.1 \mathrm{THz}$ for $\mu_{c}=0.5,0.75,1 \mathrm{eV}$, and (b) within the range $\mu_{c}=0.2 \div 1$ $\mathrm{eV}$ for $f=0.92 \mathrm{THz}$. In (a) $\hat{\beta}_{z}$ and $\hat{\alpha}_{z}$ are both represented in dashed lines for the $\mathrm{TE}_{1}$ mode and in solid lines for the $\mathrm{TM}_{2}$. In (b) $\hat{\beta}_{z}$ and $\hat{\alpha}_{z}$ are represented in solid lines and dashed lines, respectively, for both modes.

scanning over chemical potential follows an optimal quasi-linear behavior for both TE and TM modes, thus corroborating once more the tunable features of such kind of LW radiation.

As concerns plasmonic propagation, it is worth mentioning that in the TM case a SPP mode propagates in both the unbiased and the biased PW, as it always occurs between a dielectric, non-absorbing half space with $\operatorname{Re}[\varepsilon]>0$ and an adjacent conducting interface with $\operatorname{Re}[\varepsilon]<0$ [29]. The dispersion curves of this mode are shown in Fig. 6 for the GPW with a chemical potential that

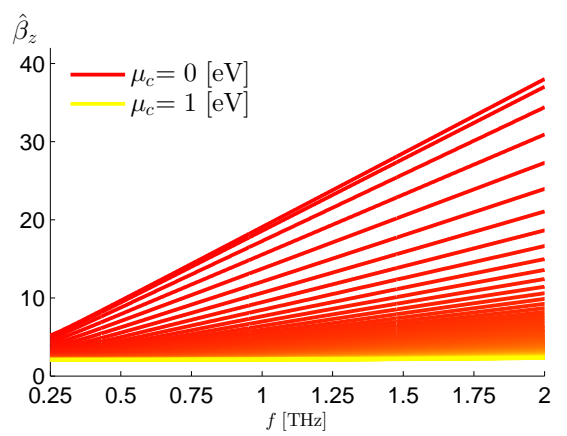

(a)

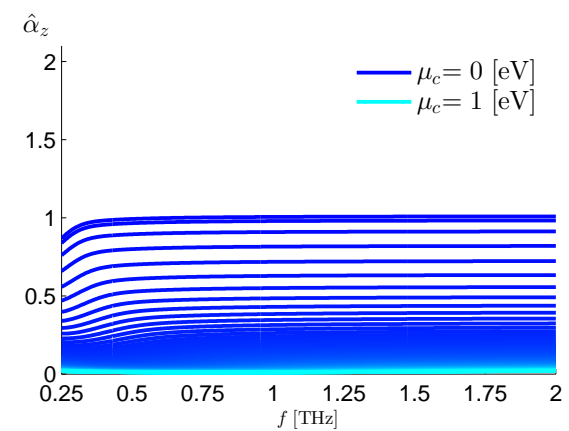

(b)

Figure 6: Dispersion curves of (a) $\hat{\beta}_{z}$, (b) $\hat{\alpha}_{z}$ for the plasmonic mode SPP. Lines become brighter (red to yellow, and blue to cyan) as $\mu_{c}$ increases from 0 to 1 $\mathrm{eV}$. Note that as $\mu_{c}$ approaches $1 \mathrm{eV}$ the SPP mode approaches the PPW TEM mode. 


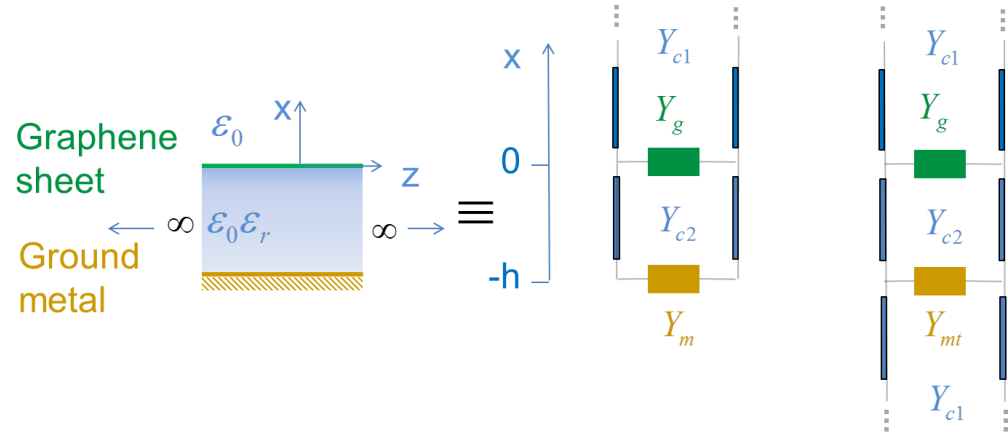

(a)

(b)

(c)

Figure 7: (a) 2D-section of the structure and its transverse equivalent networks, (b) using the approximate Leontovich boundary condition, and (c) using the transition boundary condition.

varies almost continuously from 0 to $1 \mathrm{eV}$.

\subsection{Loss Effects}

A remark about ohmic and dielectric losses at $\mathrm{THz}$ frequencies is useful. In fact, in all the results shown above, the effects of losses have been always neglected in both the dielectric substrate and the metalization. We have then computed the dispersion curves of the fundamental LWs considering a more realistic model of quartz, with a complex permittivity characterized by a real part $\operatorname{Re}\left[\varepsilon_{r}\right]=3.852$ and a loss tangent $\tan \delta=0.0141$ at $f=1 \mathrm{THz}$ [30]. On the other hand, ohmic losses have also taken into account for a metalization of gold (see Fig. $7(\mathrm{a})$ ) using two different models. The first model is related to the approximate Leontovich boundary condition [31], assuming a thick layer of gold with respect to the skin depth (see Fig. 7(b)). The equivalent admittance is then given by the formula

$$
Y_{m}=\frac{1}{1+j} \sqrt{\frac{\sigma_{m}}{\pi \mu_{0} f}}
$$

where $\sigma_{m} \simeq 44[\mu \Omega \mathrm{m}]^{-1}$ is the bulk conductivity of gold at $1 \mathrm{THz}[32]$. This value is used in place of the short circuit of the transverse equivalent network of Fig. 1. The second model does not assume a metal thickness $t$ much larger than the skin depth; however, since in any case $t \ll \lambda$, the metal layer can be modeled through a transition boundary condition (see Fig. $7(\mathrm{c})$ ). The admittance is given by the formula

$$
Y_{m t}=\sigma_{m} t
$$

where $t=150 \mathrm{~nm}$ is the thickness of the layer, and $\sigma_{m} \simeq 30[\mu \Omega \mathrm{m}]^{-1}$ is now a thin-film conductivity which is accordingly reduced of a factor 0.69 [32]. Since the skin depth of gold at $f=1 \mathrm{THz}, \delta_{\text {gold }} \simeq 127.4 \mathrm{~nm}$ is slightly shorter than 


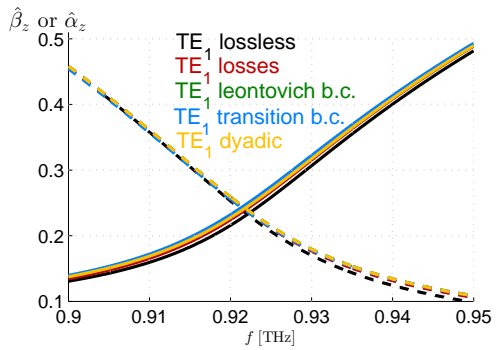

(a)

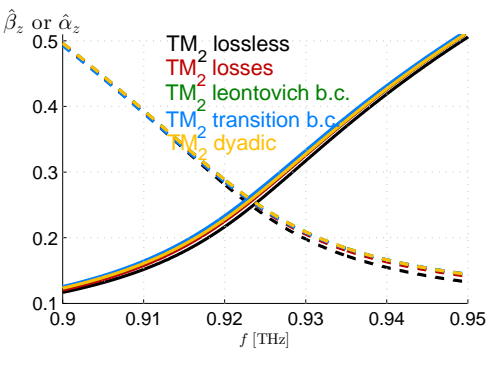

(b)

Figure 8: Effects of the introduction of losses and spatial dispersion in the curves of the fundamental LWs in the band $0.9 \div 0.95 \mathrm{THz}$ for (a) TE and (b) TM modes. The red and green-blue lines consider the effect of dielectric losses and ohmic losses, respectively. The yellow ones consider the effect of a spatially dispersive model in addition to dielectric and ohmic losses.

the thickness of the layer, the short circuit represented in Fig. 1 is now replaced by an equivalent admittance given by Eq. (8) connected to a semi-infinite transmission line of Fig. 7(c). In Figs. 8(a) and 8(b) it is clearly shown that the effect of losses is quite negligible in both TE and TM cases, and hence the ideal model previously investigated can be considered as a good approximation of more realistic conditions.

\subsection{Effects of spatial dispersion}

Furthermore, a spatially dispersive conductivity model of biased graphene [23] has been considered in order to assess the accuracy of Kubo formula. As is known [22], in the low $\mathrm{THz}$ band and for sufficiently low values of the longitudinal wavenumber $k_{z}$, spatial-dispersion effects can generally be neglected. Nevertheless, the dispersion curves of the fundamental leaky waves have been recalculated considering a non-local dyadic conductivity of graphene, that in spectral domain and in polar coordinates assumes the following form:

$$
\underline{\underline{\sigma}}=\left[\begin{array}{cc}
\sigma_{\rho} & 0 \\
0 & \sigma_{\phi}
\end{array}\right]
$$

where $\sigma_{\rho}$ and $\sigma_{\phi}$ are functions of the radial wavenumber $k_{\rho}$ only (hence graphene is isotropic) and are given by

$$
\begin{aligned}
\sigma_{\phi} & =\gamma \frac{2 \pi \alpha}{v_{F}^{2} k_{\rho}^{2}}(1-\chi) \\
\sigma_{\rho} & =\frac{v_{F}}{2 \pi \gamma_{D}(1-\chi)+v_{F} \chi} \sigma_{\phi}
\end{aligned}
$$




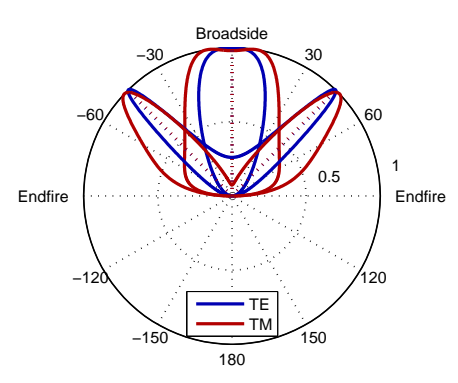

(a)

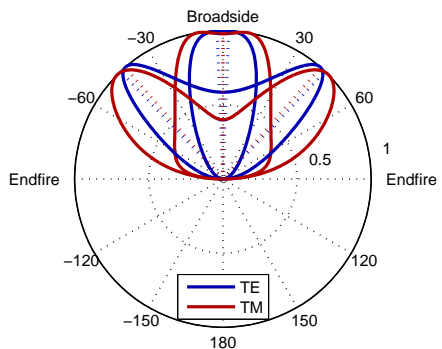

(b)

Figure 9: Radiation patterns of the fundamental TE (blue solid line) and TM (red solid line) leaky modes. Graphene conductivity is calculated with the spatially dispersive model. In (a) frequency-scanning behavior is shown for broadside $(f=0.92 \mathrm{THz})$ and $\theta=\pi / 4 \mathrm{rad}(f=0.99 \mathrm{THz})$ at $\mu=1 \mathrm{eV}$. In (b) bias-scanning behavior is shown for broadside $(\mu=1 \mathrm{eV})$ and $\theta=\pi / 4 \mathrm{rad}$ $(\mu=0.52 \mathrm{eV})$ at $f=0.92 \mathrm{THz}$.

where

$$
\begin{array}{ll}
\gamma=\frac{j q_{e}^{2} k_{B} T}{\pi^{2} \hbar^{2}} \ln \left\{2\left[1+\cosh \left(\frac{\mu_{c}}{k_{B} T}\right)\right]\right\} & \gamma_{D}=-j \frac{v_{F}}{2 \pi \omega \tau} \\
\chi=\sqrt{1-\frac{v_{F}^{2} k_{\rho}^{2}}{\alpha^{2}}} & \alpha=\omega+j \tau^{-1}
\end{array}
$$

again with electron Fermi velocity $v_{F} \simeq 10^{6} \mathrm{~m} / \mathrm{s}$. The transverse $\left(\sigma_{\phi}\right)$ conductivity affects TE fields, whereas the longitudinal one $\left(\sigma_{\rho}\right)$ affects TM fields. Therefore the value of $\sigma$ in the dispersion equations (5), (6) is respectively replaced by $\sigma_{\phi}$ and $\sigma_{\rho}$, where we have used $k_{\rho}=k_{z}$. The resulting dispersion curves are shown in Fig. 8(a) and Fig. 8(b) where they are compared with those obtained by using the Kubo formula for both the lossless and lossy case. As it can be seen, spatial dispersion has a negligible impact on the modal properties of the leaky modes (physical leaky waves are characterized by $\beta_{z}<k_{0}$ and $\alpha_{z} \leq \beta_{z}$, hence $\left|k_{z}\right|$ is of the order of $k_{0}$ and therefore spatial dispersion can be ignored [24]). As a consequence, the beam scanning over frequency and over chemical potential is almost the same, thus confirming the effectiveness of Kubo formula for a first qualitative description of the radiating properties of this kind of structures.

Finally, in Fig. 9 the far-field patterns of the fundamental TE, TM leakywave modes are calculated through a standard application of the reciprocity theorem [33], considering for simplicity a $2 \mathrm{D}$ case in which the excitation is an ideal electric line source placed in the middle of the substrate (TE), or a magnetic line source placed on the ground plane (TM). In Fig. 9(a) the beamscanning behavior is obtained by varying the frequency while keeping $\mu_{c}=1$ eV, whereas in Fig. 9(b) the beam-scanning behavior is obtained by varying 
the chemical potential at the fixed frequency $f=0.92 \mathrm{THz}$. In both figures a comparison between TE, TM patterns is shown for two different pointing elevation angles $(\theta=0, \pi / 4 \mathrm{rad})$. As expected, the pointing angle is reached at almost the same frequencies (see Fig. 9(a)) or bias (see Fig. 9(b)), since both the phase and the attenuation constants are equally affected. It is worth here to notice that the equalization of the TM, TE leaky-wave phase constants allows for both frequency- and bias-scanning for a considerable range of the elevation angle with a nearly circular conical scanned beam [34].

\subsection{Technological device implementation}

In this subsection we aim to provide some technological details about the practical realization of a GPW. The proposed structure is depicted in Fig. 1 where the antenna feed excitation can be achieved through a finite-size slot etched in the ground plane. The structure must be electrically large in the transverse directions, in order to minimize edge effects on the shape of the radiation patterns. In particular, considering a circular substrate with radius $\rho_{a p}$ and assuming, as is customary, that the relevant leaky modes have radiated $90 \%$ of their power before reaching the structure edges, it can readily be shown that

$$
\frac{\rho_{a p}}{\lambda_{0}} \simeq \frac{0.18}{\hat{\alpha}_{z}}
$$

where $\lambda_{0}$ is the free-space wavelength at $f=1 \mathrm{THz}$. With reference to the case illustrated in Fig. 5, where $\hat{\alpha}_{z} \simeq 0.2$, this implies a substrate having linear dimensions of the order of $500 \mu \mathrm{m}$. However, such dimensions are nowadays well within the state of the art for the production of high-quality graphene sheets, thanks to the recent advances in chemical vapor deposition (CVD) [35]. Just recently, a large-size and high-quality single-layer graphene film has been synthesized on copper foils by chemical vapor deposition using methane [36].

Once the graphene film is produced, it can be transferred onto a backmetalized $\mathrm{SiO}_{2}$ substrate (already comprising the polysilicon layer) using the polymethyl methacrylate (PMMA) based wet chemical process [35]. Ultimately, a coherent $\mathrm{THz}$ source, such as a QCL beam, can be used to illuminate a slot etched in the metalized back plane of the antenna in order to suitably excite the fundamental leaky modes. The tunable features of graphene are then exploited by varying the $\mathrm{DC}$ voltage between the graphene sheet and the polysilicon layer, used here as a gate electrode.

\section{Conclusion}

We have reported a detailed dispersion analysis of a graphene-based planar waveguide (PW) over the frequency range $0.25-2 \mathrm{THz}$ considering also the application of a chemical potential $\mu_{c}$ in the range from 0 to $1 \mathrm{eV}$. We have compared the modal spectrum of this structure to the grounded dielectric slab (GDS) and to the parallel-plate waveguide (PPW) ones, finding that the modes 
of the unbiased graphene are much closer to the GDS modes and approach the PPW modes as the chemical potential approaches $1 \mathrm{eV}$. As a consequence the tunability of graphene allows for switching the graphene-based PW from GDS to PPW. However, the main result emerging from our dispersion analysis is represented by the presence of the fundamental leaky modes for both the TE and TM polarizations. It is seen that for $\mu_{c}=1 \mathrm{eV}$ these modes exhibit suitable leakage rates, opening very interesting perspectives for antenna applications. In particular, the tunability of graphene allows for beam scanning functionalities

at a fixed frequency, a feature that could be of great interest in the context of reconfigurable $\mathrm{THz}$ antennas. Future work can be devoted to deepen the study of the radiation properties of our graphene-based PW taking also into account a more realistic dipole-like source and a suitable biasing scheme.

\section{References}

[1] A. K. Geim and K. S. Novoselov, Nat. Mater. 6(3), 183 (2007).

[2] P. H. Siegel, IEEE Trans. Microw. Theory Tech. 50(3), 910 (2002).

[3] G. W. Hanson, J. Appl. Phys. 103(6), 064302 (2008).

[4] A. Vakil and N. Engheta, Science 332(6035), 1291 (2011).

[5] F. Schweirz, Nat. Nanotechnol. 5(7), 486 (2010).

[6] M. Dragoman, A. A. Muller, D. Dragoman, F. Coccetti, and R. Plana, J. Appl. Phys. 107(10), 104313 (2010).

[7] M. Jablan, H. Buljan, and M. Soljacic, Phys. Rev. B 80(24), 245435 (2009).

[8] M. Tamagnone, J. S. Gómez-Diaz, J. R. Mosig, and J. Perruisseau-Carrier, J. Appl. Phys. 112(11), 114915 (2012).

[9] J. Perruisseau-Carrier, M. Tamagnone, J. S. Gómez-Diaz, M. EsquiusMorote, and J. R. Mosig, Proceedings of IEEE Antennas and Propagation Society International Symposium (AP-S/URSI), Orlando, pp. 136-137 (2013).

[10] R. Filter, M. Farhat, M. Steglich, R. Aalae, C. Rockstuhl, and F. Lederer, Optics Express 21(3), 3737 (2013).

[11] M. Esquius-Morote, J. S. Gómez-Diaz, and J. Perruisseau-Carrier, IEEE Trans. Terahertz Sci. Technol. 4(1), 116 (2014).

[12] X.-C. Wang, W.-S. Zhao, J. Hu, and W.-Y. Yin, IEEE Trans. Nanotechnol. $14(1), 62(2015)$.

[13] T. Tamir and A. A. Oliner, Proc. Inst. Electr. Eng. 110(2), 310 (1963). 
[14] A. A. Oliner and D. R. Jackson, in Antenna Engineering Handbook, ed. by J. L. Volakis (McGraw-Hill, New York, 2007), Ch. 11.

[15] Z. G. Liu, J. Infrared Millim. Terahertz Waves 31(4), 391 (2010).

[16] N. Marcuvitz, Waveguide Handbook, (McGraw-Hill, New York, 1951).

[17] L. Felsen and N. Marcuvitz, Radiation and Scattering of Waves, (Englewood Cliffs, Prentice-Hall, 1973).

[18] R. Sorrentino, in Numerical Techniques for Microwave and Millimeter-wave Passive Structures, ed. by T. Itoh (Wiley, New York, 1989), Ch. 11.

[19] G. Valerio, D. R. Jackson, and A. Galli, IEEE Trans. Microw. Theory Tech. 58(7), 1786 (2010).

[20] G. Lovat, P. Burghignoli, and D. R. Jackson, IEEE Trans. Antennas Propag. 54(5), 1442 (2006).

[21] V. P. Gusynin, S. G. Sharapov, and J. B. Charbotte, New J. Phys. 11(9), 095013 (2009).

[22] G. W. Hanson, IEEE Trans. Antennas Propag. 56(3), 747 (2008).

[23] G. Lovat, G. W. Hanson, R. Araneo, and P. Burghignoli, Phys. Rev. B 87(11), 115429 (2013).

[24] G. Lovat, P. Burghignoli, and R. Araneo, IEEE Trans. Electromagn. Compat. 55(2), 328 (2013).

[25] G. Valerio, D. R. Jackson, and A. Galli, Proc. Royal Soc. A 466(2120), 2447 (2010).

[26] V. Galdi and I. M. Pinto, Microw. and Opt. Tech. Lett. 24(2), 135 (2000).

[27] P. Lampariello, F. Frezza, and A. A. Oliner, IEEE Trans. Microw. Theory Tech. 38(12), 1831 (1990).

[28] C. Di Nallo, F. Frezza, A. Galli, P. Lampariello, and A. A. Oliner, IEEE Trans. Microw. Theory Tech. 42(12), 2429 (1994).

[29] S. A. Maier, Plasmonics: Fundamentals and applications (Springer, New York, 2007).

[30] M. Naftaly and R. E. Miles, IEEE Proc. 95(8), 1658 (2007).

[31] D. M. Pozar, Microwave Engineering (Wiley, 1997).

[32] N. Laman and D. Grischkowski, Appl. Phys. Lett. 93(5), 051105 (2008).

[33] T. Zhao, D. R. Jackson, J. T. Williams, and A. A. Oliner, IEEE Trans. Antennas Propag. 53(11), 3525 (2005). 
[34] P. Baccarelli, P. Burghignoli, F. Frezza, A. Galli, P. Lampariello, G. Lovat, and S. Paulotto, IEEE Trans. Microw. Theory Tech. 53(1), 32 (2005).

[35] G. Deokar, J. Avila, I. Razado-Colambo, J.-L. Codron, C. Boyaval, E. Galopin, M.-C. Asensio, and D. Vignaud, Carbon 89, 82 (2015).

[36] X. Li, W. Cai, J. An, S. Kim, J. Nah, D. Yang, et al., Science 324(5932), $1312(2009)$. 